Meta

Journal des traducteurs

Translators' Journal

\title{
Terminologie et efficacité de la communication : critères linguistiques
}

\section{Rostislav Kocourek}

Volume 30, numéro 2, juin 1985

URI : https://id.erudit.org/iderudit/003331ar

DOI : https://doi.org/10.7202/003331ar

Aller au sommaire du numéro

Éditeur(s)

Les Presses de l'Université de Montréal

ISSN

0026-0452 (imprimé)

1492-1421 (numérique)

Découvrir la revue

Citer cet article

Kocourek, R. (1985). Terminologie et efficacité de la communication : critères

linguistiques. Meta, 30(2), 119-128. https://doi.org/10.7202/003331ar d'utilisation que vous pouvez consulter en ligne.

https://apropos.erudit.org/fr/usagers/politique-dutilisation/ 


\title{
TERMINOLOGIE ET EFFICACITÉ DE LA COMMUNICATION : CRITĖRES LINGUISTIQUES
}

\author{
ROSTISLAV KOCOUREK
}

La tâche principale de la langue de spécialité est d'exprimer et de communiquer le contenu spécialisé. Dans le présent article, il ne s'agira donc pas de la théorie de la communication et de la théorie statistique de l'information, qui font abstraction du contenu des messages et où l'efficacité est le complément de la redondance.

Il s'agira de la communication humaine, sémantique, qualitative, qui met l'accent sur le contenu et sur la forme linguistique du message, et qui présuppose que l'émission, le transfert et la réception de la forme du message se déroule techniquement d'une manière satisfaisante. Il s'agira du transfert de l'information au moyen de messages (Guiraud $1968: 145$ ).

Linguistiquement, message veut dire texte parlé ou écrit (discours), qui a une forme (un signifiant) et un contenu (sens, signifié, information). Le texte parlé ou écrit est fondé sur le système de la langue, que l'on suppose connu à la fois de l'auteur et du destinataire.

Si l'on mettait l'accent sur le côté pragmatique ou psycholinguistique de la communication, on soulignerait le rôle des participants : le rôle de l'auteur, qui exprime le contenu spécialisé au moyen de la forme d'un texte ; le rôle du destinataire, qui perçoit la forme du texte et qui en comprend le contenu.

Mais nous aborderons le problème d'un côté linguistique : nous dirons que l'efficacité de la communication doit être donnée par diverses qualités linguistiques du texte et du système linguistique qui le sous-tend. Ces qualités devraient évidemment être pertinentes pour l'expression, pour la perception et pour la compréhension du texte.

Reste à observer que le sujet du présent article n'est pas le rapport entre la communication et les textes, mais celui entre la communication et la terminologie (ensemble de termes). Cela pose le problème si l'on peut évaluer la communication spécialisée en se concentrant sur les unités lexicales (termes) et non sur la syntaxe des textes.

Du point de vue sémantique, les termes (mots et syntagmes lexicaux) sont des unités primordiales des textes et du système de la langue spécialisée. Du point de vue grammatical, il existe des écoles de pensée linguistique qui accèdent aux phrases et aux textes par les unités lexicales, prétendant que la structure de la phrase est conditionnée par la structure du mot - par son sens et par ses qualités grammaticales (signification conceptuelle et relationnelle).

Il est donc légitime - bien que cela ne soit pas la seule approche possible, et sans mésestimer le rôle capital de la syntaxe phrastique et textuelle - de s'attendre à des résultats intéressants si on examine les termes, plutôt que les phrases, du point de vue de l'efficacité de la communication spécialisée. 
Pour nous, dans cet article, l'efficacité de la communication spécialisée ne sera définie qu'indirectement par diverses qualités linguistiques - textuelles et systémiques des termes. Il s'agira, on l'a noté, des qualités des termes ayant trait, d'une part, à l'expression et à la perception de la forme parlée ou écrite des termes et, d'autre part, à la compréhension de leur sens.

Pour pouvoir apprécier l'efficacité des termes, nous sommes obligés de chercher les critères d'évaluation de la terminologie. Les formulations des critères d'évaluation ne manquent pas dans l'histoire de la terminologie ; il y a par exemple l'exposé, de 1787, des principes de la nomenclature chimique de Guyton de Morveau (Fuchs 1966 : $652-653$, Guiraud $1978: 73-74)$ et à une date plus récente, la morale terminologique de C.S. Peirce (1978: 61 sq.).

Dans la littérature terminologique contemporaine, on explore souvent les qualités idéales des termes du point de vue d'une normalisation éventuelle. On parle d'indices de la bonne qualité linguistique (Güteeigenschaften der Sprache, Wüster 1970:85-122, surtout p. 86), de principes terminologiques (Mazur 1961), de critères de terminologie (Horecký 1965), d'exigences à l'égard des termes (ISO 1968), de critères d'acceptabilité des néonymes (Auger et Rousseau 1978: 58-59), de critères de sélection terminologique (Kocourek 1980 : 1172), et de critères de choix de néonymes (Rondeau 1981 : 130-135).

Afin de pouvoir mieux comprendre les conditions d'ordre terminologique de l'efficacité de la communication, nous examinerons les critères disponibles, et proposerons certains critères nouveaux (voir Kocourek 1982: 196-200). Nous allons procéder d'un plan linguistique à l'autre : des critères graphico-phoniques et grammaticaux aux critères lexicaux, et nous nous limiterons à la langue standard.

Sur le plan phonique et graphique, les formes parlée et écrite du terme peuvent être évaluées du point de vue de leur durée ou étendue, jugées acceptables ou non. On parle de la brièveté, de la concision, de l'économie du terme, que l'on pourrait définir, par exemple, comme le nombre de phonèmes, de lettres et d'autres signes orthographiques, de syllabes, de morphèmes, de mots.

La communication est-elle plus efficace si on emploie le terme boisson gazeuse, ou son synonyme soda (angl. soft drink, Répertoire 1982 : 11 et Répertoire 1983)? Le terme bloc obturateur de puits est-il plus efficace que le terme B.O.P. ? Le terme tige d'entraînement est-il préférable au terme kelly (Néologie en marche $1975: 7$ ) ?

L'idéal de la concision terminologique en langue spécialisée est souvent en concurrence avec le désir de refléter, par la forme, le contenu complexe et riche du terme. Le résultat extrême de ce désir est la création des termes constitués par des syntagmesfleuves très descriptifs et proches de la définition.

L'une des causes du succès de l'abréviation et de l'emprunt à l'anglais est leur concision : Cedex l'emporte sur courrier d'entreprise à distribution exceptionnelle, zoom sur objectif à distance focale variable.

Un autre critère sur le plan de la deuxième articulation pourrait être la facilité phonique (de prononciation ou de perception auditive) et la facilité graphique (d'orthographe ou de lecture). La facilité, commodité, simplicité graphico-phonique n'est pas un caractère fondamental, mais son absence peut contribuer au rejet d'un terme ; des termes comme phtisie [ftizi] (angl. et lat. phthisis) sont souvent remplacés par des termes de facilité graphico-phonique supérieure. On essaie parfois même de chercher l'euphonie du terme.

Un critère apparenté au précédent est le degré de conformité aux structures phoniques et graphiques de la langue donnée. Le terme concerné, un emprunt surtout, est-il conforme aux éléments et aux enchaînements de la structure phonologique et de la structure graphique de la langue? Afin de pouvoir être admis au lexique français et uti- 
lisés sans gêne dans les textes français, les termes étrangers sont plus ou moins adaptés au système de la langue française. On parle de l'intégration, de l'assimilation phonique et graphique : gas-oil [, ], gazole []. Toutes choses égales d'ailleurs, une forme intégrée peut probablement mieux réaliser sa fonction de communication.

Ce critère graphico-phonique fait partie de ce qu'on appelle conformité ou " propriété » linguistique (langagière), c'est-à-dire la conformité aux caractères et aux règles de la langue, au génie de la langue. La conformité linguistique est plus importante encore sur les autres plans de la langue.

Quant à la morphologie flexionnelle, il s'agit de catégories grammaticales et de morphèmes flexionnels du terme, qui doivent être déterminés, examinés et évalués par rapport aux autres unités lexicales de la langue. Dans le cas des emprunts, l'incapacité d'un élément allogène de former les variantes flexionnelles exigées en langue emprunteuse constitue un obstacle d'intégration. On pensera à la difficulté, en français, d'assimiler les verbes anglais. On trouve, en langue standard, check-up pour bilan de santé, mais non le verbe correspondant.

Pour ce qui touche à la syntaxe, le terme doit pouvoir accomplir les fonctions syntaxiques de sa partie du discours (classe lexicale). Il est surtout question de termes syntagmes, si nombreux en terminologie : la maniabilité syntaxique est un des critères de leur efficacité dans la communication. La maniabilité syntaxique du terme liquidation des droits (liquidation de retraite, angl. pension calculation) sera syntaxiquement mieux maniable que le terme régime salaire meilleures années (angl. average best earnings plan), mais moins maniable que liquidation (Répertoire 1982:48). Maints néonymes proposés échouent au test de maniabilité syntaxique au sein du texte.

C'est sur le plan lexical que l'on peut situer la plupart des critères disponibles : sur le plan de morphèmes non flexionnels, de mots et de syntagmes lexicaux. Exprimé d'une manière générale, le problème est celui de la compatibilité lexicale du terme avec d'autres unités du lexique ; on préfère, pour un concept donné, une unité lexicale - morphème(s), mot(s), syntagme - ayant sa place dans le système lexical de la langue, c'està-dire pourvue de qualités et de rapports lexicaux désirés, et dépourvue de qualités et de rapports lexicaux non désirés.

À l'intérieur du système terminologique donné, il s'agit en premier lieu de la systémicité du terme : de ses rapports aux cohyponymes, aux hyponymes, à l'hyperonyme. Puisque l'on présuppose un système conceptuel structuré, il est important que la forme du terme soit différente de celle des autres termes du système. Bien que cela ne soit pas nécessaire, on considère ordinairement bénéfique pour la communication si la forme du terme indique les oppositions pertinentes, et rien qu'elles, et si elle reflète le degré de différence entre les concepts désignés. Ce qui est essentiel c'est ce que Eugen Wüster nommait distinctivité (all. Unterscheidbarkeit), qui est à la base de toute systémicité.

Le terme appartient d'abord à un type particulier de formation lexicale et, si l'on compare deux termes, n'importe quel type de formation peut être en apposition avec n'importe quel autre. Ceci a une importance considérable dans l'évaluation des termes du point de vue non seulement de la normalisation terminologique mais également de la communication efficace. Ne veut-on pas normaliser surtout afin d'augmenter l'efficacité de la communication?

Quelle est la valeur respective de différents types de formation du point de vue des objectifs visés ? La dérivation impropre, régressive et propre (affixation), la confixation gréco-latine (formation savante), la composition, la lexicalisation de syntagmes, l'emprunt, le calque, l'abréviation, l'emploi figuré, l'emploi de termes arbitraires - ces types de formation sont-ils tous acceptables (cf. Kocourek 1982 : 154) ? Si oui, dans quelle mesure, dans quel ordre de priorité et dans quels cas ? 
C'est là un exemple concret de l'impossibilité de déterminer les critères absolus de l'efficacité de la communication spécialisée. Notre évaluation des types de formation peut, en effet, varier en fonction de l'objectif visé, du domaine, de l'école de pensée, de l'entreprise, du niveau d'intellectualisation, du registre de langue, etc. Et cette variation n'est pas nécessairement un caprice individuel, mais une réponse aux exigences fonctionnelles ou, si l'on préfère, aux besoins qui déterminent l'efficacité de la communication.

Un administrateur, un juriste, un professeur peut préférer la dérivation, un médecin la confixation, un mathématicien l'emploi spécial de mots usuels, un ingénieur qui veut être compris sur le chantier - un emprunt, même peu intégré, mais connu de tout le monde.

Un autre aspect lié à la formation du terme est sa productivité, son caractère formateur, sa dérivabilité, sa reproductibilité, la capacité de sa racine de former les dérivés. La facilité de la communication peut souvent augmenter s'il est possible de former, à partir d'un nom par exemple, d'autres noms, adjectifs et verbes nécessaires. On appelle homogénéité de la terminologie l'idéal de former tous les termes conceptuellement apparentés à partir de la même racine.

L'abus de la productivité peut cependant devenir un obstacle à la compréhension rapide; les paires de termes trop apparentés comme signifiant/signifié, référencel référent, destinateur/destinataire, allocutoire/illocutoire (ou perlocutoire), parascolaire/ périscolaire (Répertoire $1983: 3$ ) peuvent gêner plutôt que faciliter la communication.

On ne peut ne pas aborder, dans cet ordre d'idées, le problème de l'origine du terme et de son rapport aux termes employés dans d'autres langues. Quel rôle joue l'origine du terme dans la communication efficace? Doit-on toujours forger des termes français autochtones, ou faut-il parfois choisir les emprunts ? La confixation gréco-latine est-elle préférable à la formation française (cenomètre vs pèse-vin), ou à l'emprunt à l'anglais (oléoduc vs pipeline)?

Dans quel sens et dans quelle mesure devons-nous tenir compte de l'aspect interlinguistique du terme (concept vs notion) ? Comment faire face au conflit possible entre les exigences de l'intégrité de la langue, les besoins du bilinguisme, et les besoins du contact entre plusieurs langues?

Nous vivons dans un monde où le contact des langues est habituel et non exceptionnel. Il paraît donc que - pour augmenter l'efficacité terminologique — on devrait essayer de contribuer à l'harmonie interlinguistique des termes tout en respectant l'intégrité et la structure de la langue naturelle donnée.

Le problème de la communication humaine est étroitement lié au groupe social : communication efficace au sein de quel groupe ? Le groupe peut dépasser le groupe de travail, l'entreprise, l'organisme plus large, la communauté régionale et même nationale.

Revenons encore à l'origine des termes. La volonté, ou l'absence de volonté, d'accepter un terme d'origine étrangère comme moyen de communication nous mène à une particularité importante de la communication. Les schémas linguistiques usuels de la communication humaine soulignent la fonction cognitive, le faire-savoir, le transfert du contenu, de l'information. Ces schémas présupposent un accord idéal ou satisfaisant entre l'auteur et le destinataire. Mais, pour communiquer vraiment, il faut vouloir accepter ou faire accepter, il faut que le contenu communiqué soit accepté, c'est-à-dire non seulement reçu mais effectivement assumé (Greimas et Courtés 1979 : 47-48).

C'est que l'efficacité de la communication peut être gênée par l'attitude émotive du destinataire envers, par exemple, l'origine des termes employés. La communication d'un contenu cognitif peut être moins efficace si le destinataire n'est pas prêt à accepter 
la terminologie, ou bien parce qu'elle fourmille de xénismes ou bien parce qu'elle est pleine de néonymes français peu connus du destinatäire.

Quoique nous n'envisagions ici que la langue spécialisée standard (soignée et courante), il s'impose de noter que ces difficultés du faire-accepter sont aggravées par la différenciation sociale et fonctionnelle de la langue tout entière (langue standard et non standard, langue spécialisée et usuelle, divers registres de la langue non standard).

Nous arrivons aux critères lexicaux d'ordre sémantique. Nombre de questions sont liées aux interférences sémantiques du terme que l'on observe.

Ce terme possède-t-il, dans la langue, des paronymes gênants ? - Conatif pourrait être compromis par cognitif, idiolecte par idiot (de la même origine mais sémantiquement très éloigné).

Le sens suggéré ou étymologique des morphèmes constitutifs est-il acceptable ?Par exemple, le terme philologie était probablement abandonné parce que les protagonistes de l'étude nouvelle de la langue se voulaient scientifiques plutôt qu'amateurs de lettres ou de mots.

Les acceptions non terminologiques des mots terminogènes sont-elles conformes aux besoins et aux préférences des spécialistes ? - Les générativistes nommaient structure superficielle ce que d'autres linguistes considéraient comme point de départ important, et non superficiel, de l'analyse syntaxique. On dit acadien, ou langue acadienne plutôt que dialecte acadien, et on a rejeté, comme péjorative, l'expression patois. cation.

Dans tous ces cas d'interférences, il peut y avoir une certaine gêne de la communi-

L'exemple de philologie nous montre l'importance de la motivation, qui constitue le côté sémantique de la formation lexicale. Le sens des unités lexicales de la langue est donné, en fin de compte, par la convention. Mais la majorité des unités lexicales sont motivées, et non arbitraires (immotivées). En terminologie, la prédominance du motivé est si prononcée qu'elle est son caractère essentiel (cf. Guiraud 1978: 98). La forme des termes suggère souvent une partie de leur sens. Ceci découle non seulement de la nature du lexique en général, mais aussi de l'intellectualisation de la langue spécialisée, surtout technoscientifique. Les scientifiques créent des termes en donnant, normalement, une explication rationnelle de la forme choisie. Les terminologues examinent la motivation des termes et sa justification. La motivation des termes est donc un aspect important de l'étude des termes.

Certains critères d'évaluation sont liés au rapport motif/sens des termes. Il y a motivation si la forme du terme peut suggérer des éléments du contenu qui indiquent pourquoi la forme est employée pour désigner le concept (le sens exprimé par la définition). Le motif, c'est le contenu motivationnel du terme, ce sont les éléments de contenu, suggérés par les morphèmes du terme et qui expliquent pourquoi la forme est utilisée (cf. Kocourek 1982 : 156-157).

La place du motif dans l'étude du terme en tant que signe peut être indiquée schématiquement ainsi :

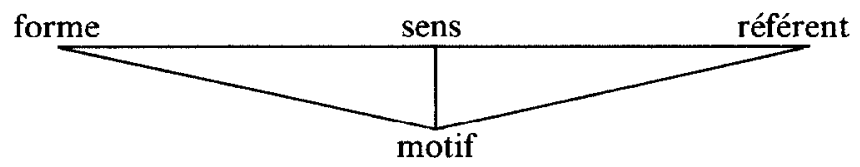

On peut se poser la question suivante : le motif correspond-il au sens? - Par exemple, le motif du terme ambigu est " ayant deux significations ", tandis que son sens, dans cet exposé, est " ayant la même forme et deux ou plusieurs acceptions ou sens différents ? (= "étant polysémique ou homonymique »). Il y a donc une certaine diver- 
gence entre le motif et le sens de ambigu. Son motif est plus étroit que son sens. Il est, par conséquent, légèrement trompeur, puisqu'il suggère le sème de "deux " au lieu de "deux ou plusieurs" acceptions ou sens.

Faut-il accepter cette divergence motif/sens, ou faut-il remplacer ambigu par une autre forme ? On sait que, en fin de compte, c'est la convention qui décide, et qui peut, en effet, consacrer un terme dont la motivation est douteuse.

L'efficacité de la communication exige une convention fixée (voir stabilité et usage, plus bas), mais elle peut être renforcée par une motivation acceptable.

Afin de faciliter la communication, il est utile d'employer, de créer, de normaliser les termes qui ne suggèrent pas une idée fausse ou, si possible, qui orientent correctement. On a essayé, sans grand succès toutefois, de remplacer, pour le sens " signe graphique symbolisant un morphème", le terme idéogramme (motif : "signe graphique symbolisant une idée ") par le terme un peu mieux motivé logogramme (motif : "signe graphique symbolisant un mot "). On essaie d'introduire le terme langue standard pour remplacer le terme langue littéraire, qui fait trop penser à la langue écrite de la littérature.

Les études terminologiques sont riches en discussions relatives à la motivation dite sémantique des termes. Est-il préférable d'employer un terme dont la motivation est donnée par une métaphore terminologique (cf. Kocourek 1982 : 147-149), ou par des caractères descriptifs (inhérents ou intrinsègues, ex. : forme, matériau, couleur, dimension) ou par des caractères relationnels (extrinsèques : de destination, ex. : emploi, place, fonctionnement, ou de provenance, ex. : producteur, inventeur, fournisseur, méthode de production, pays d'origine, époque ; cf. Kocourek 1982 : 149-151) ? Ce sont les Ordnungsmerkmale de Wüster (1979 : 14-19), qui sont importants en didactique de la terminologie et, indirectement, pour l'efficacité de la communication spécialisée.

L'aspect didactique suscite les questions suivantes : le motif indique-t-il clairement le sens, c'est-à-dire le terme est-il transparent, " logique", clair ? Par exemple, lequel des deux synonymes (pour le sens " période de temps pour laquelle sont établies des prévisions financières dans un organisme public ") est plus transparent : exercice financier ou année financière (angl. fiscal year, Répertoire $1982: 58$ ) ?

Il existe plusieurs critères psycholinguistiques qui touchent à cet aspect des termes : le pouvoir d'évocation, la facilité de mémorisation, la compréhensibilité, et surtout la lisibilité des textes.

Il est notoire que les textes spécialisés sont de mauvaise lisibilité pour les noninitiés. Tous les indices de lisibilité comprennent, outre la composante syntaxique, une composante lexicale, terminologique (Richaudeau 1973 : 89-97).

La formule de Dale-Chall prend en considération le Dale-score, c'est-à-dire le pourcentage de mots difficiles, extérieurs à une liste type de 3000 mots dont $80 \%$ au moins sont connus des enfants de la classe 4.

Toutes les deux cotes de Rudolf Flesch, la facilité de lecture (reading ease) et l'intérêt humain, prennent en considération la longueur de mots en syllabes ( $W=$ le nombre moyen de syllabes par 100 mots).

L'une des deux variables de la cote brouillard (fog index) de R. Gunning est le pourcentage de mots de plus de 3 syllabes.

Selon F. Richaudeau, les mots courts favorisent la mémorisation et donc la lisibilité ; la longueur de mots est donnée en lettres par mots (moins de 4 lettres; entre 4 et 4,3 lettres; entre 4,3 et 4,6 lettres ; plus de 4,6 lettres).

Selon Funkhouser et Maccoby $(1971: 68)$, un grand nombre de mots difficiles et scientifiques défavorise le plaisir retiré de la lecture et l'attitude positive du lecteur, après la lecture, envers la spécialité du texte. 
On voit que, en ce qui concerne les non-initiés, les mots difficiles, longs, scientifiques défavorisent la communication efficace du contenu. Mais les spécialistes, eux, sont des initiés. Ils construisent ou apprennent leur système terminologique afin de saisir les choses de leur spécialité, afin de concevoir et de manier les concepts nécessaires, et de faire progresser et de communiquer la connaissance du domaine. Dire que la terminologie et son apprentissage sont difficiles c'est dire que le processus même de la connaissance et la communication de la connaissance sont difficiles.

Les études de lisibilité pourraient néanmoins nous rappeler de ne pas écarter le critère de la concision terminologique (ci-dessus).

La tâche principale de la terminologie, c'est de signifier les concepts et d'aider à communiquer les concepts dans le cadre de la communication spécialisée. Le terme est donné par la définition spécialisée où le défini est la forme du terme et où le syntagme définissant est le sens du terme (concept, notion).

La définition délimite le concept donné, elle le met en rapport avec les termes apparentés, le situe dans le système terminologique dont il est question. Comprendre un terme, c'est saisir sa définition. La communication spécialisée ne peut pas être efficace sans une précision sémantique des termes, indiquée par une définition connue des usagers.

En plus, la définition d'un terme par des spécialistes présuppose déjà ce que l'on appelle la nécessité du terme, ou sa correspondance aux besoins des spécialistes.

On pourrait aller plus loin, au-delà du rapport forme/sens du terme. Le terminologue-linguiste préfere, cependant, ne pas explorer, ni mettre en question la justification du terme et d'autres aspects du rapport sens-référent. La communication des connaissances spécialisées, au sein même de la communauté des spécialistes, peut être dérangée par l'introduction de concepts peu justifiés (et par l'exclusion de concepts indispensables). On a essayé d'introduire dans les études terminologiques le concept de l'exactitude, dont on parle si le sens correspond au référent du terme (cf. Rondeau éd. 1979 : 152-153). Remarquons en marge que le terme definiteness a été proposé pour couvrir la combinaison de l'exactitude et de la biunivocité.

L'idéal de la biunivocité des termes (Wüster 1970 : 94) ne peut pas manquer dans l'évaluation terminologique. La biunivocité, c'est l'univocité et la dénomination unique (Kocourek $1979: 151-154$ et $1982: 199$ ).

Prenons d'abord l'univocité. C'est l'absence de l'ambiguité (de l'homonymie et de la polysémie); on l'appelle aussi monosémie ou monovalence. C'est l'existence d'un seul concept pour une forme donnée. Linguistiquement parlant l'univocité présuppose la neutralisation de l'homonymie et de la polysémie, c'est-à-dire de l'ambiguïté.

Dans les textes spécialisés, le danger de la collision homonymique est généralement réduit grâce à la restriction du texte à un domaine spécialisé. En réalité, on ne peut pas vraiment s'attendre à ce que, dans un texte parlé d'une spécialité donnée, les membres de la série homonymique [] par exemple entrent en collision : air, aire, ère, erre, ers, hère, haire, $R, r, / r /$, Aire, etc. On voit aussi que le texte écrit élimine bien des collisions possibles en langue parlée.

Ce qui est beaucoup plus grave, c'est le danger de la collision polysémique : un spécialiste peut redéfinir un terme, employer la même forme pour un concept différent. D'importants désordres de communication peuvent en résulter ; on pensera aux dégâts que peut causer la polysémie des termes comme structure (par exemple la structure des structuralistes et la structure des générativistes), langue (la langue de Saussure, la langue de l'école de Prague) et morphème (celui de Bloomfield, celui de Martinet). Le même phénomène existe dans toutes les spécialités. C'est encore à cause de la polysémie 
terminologique que la maîtrise et le respect de la définition des termes sont jugés essentiels pour une communication efficace.

L'homonymie et la polysémie, ne l'oublions pas, sont des principes universels des langues, liés à leur économie. Elles ne vont pas disparaître parce qu'on les interdit ou qu'on les supprime. Tout en essayant de réduire le nombre d'expressions homonymes et la polysémie des termes, on ne va pas prétendre que les phénomènes n'existent pas en langue de spécialité (cf. Kocourek 1982 : 162).

Certains couples homonymiques et différentes acceptions données par plusieurs définitions non synonymes sont inévitables et peuvent bien servir une communication efficace si on les emploie selon les règles de communication non ambiguë. Il nous semble que, dans le système terminologique, l'idéal c'est l'homonymie et la polysémie réduites, limitées aux cas nécessités par les exigences de la communication. Dans les textes, toute collision homonymique et polysémique doit être évitée ou clairement signalée. Les textes doivent être désambiguïsés.

Passons maintenant à l'autre composante de la biunivocité : à la dénomination unique (ou l'absence de synonymie, de polylexie, de polymorphisme; voir Kocourek 1982:166).

De même que l'ambiguïté, la synonymie lexicale est réduite si on ne tient compte que des termes qui appartiennent à un domaine et à un niveau d'intellectualisation donné. Comme l'ambiguïté, la synonymie est un principe universel des langues : elle peut être analysée, aménagée, réduite, mais non éliminée.

Manuila et al. (1970-1975, vol. $3: 730$ ) indiquent 27 synonymes du terme splénomégalie myéloïde idiopathique, dont anémie myélophtisique et érythroblastose chronique de l'adulte. Sans nous ingénier, nous avons repéré 27 dénominations pour le concept du "groupe de mots lexicalisé " (Kocourek 1979 : 124).

Au sein du système, la synonymie peut être limitée sans toutefois fermer la voie par laquelle s'effectuent les recherches et les applications nouvelles. Dans les textes, toute collision synonymique, comme la collision homonymique et polysémique, doit être évitée ou clairement signalée.

Remarquons que, dans notre texte, tout en préférant certains termes, nous signalons des synonymes ou des séries synonymiques correspondant à la variété d'approches de différents chercheurs.

Nous avons signalé ailleurs (Kocourek $1983: 257-258$ ) que l'idéal de biunivocité est complémenté par l'idéal de la sauvegarde de la multiplicité et de la flexibilité d'approches, l'idéal du respect de la pluralité des fonctions et de la liberté d'expérimentation individuelle, qui permettent d'assurer la connaissance objective et applicable. Ces idéaux de variété, eux aussi, ont besoin d'une communication efficace, ce qui est à l'origine des séries synonymiques.

Le dernier critère d'évaluation que nous allons mentionner, c'est l'usage du terme, sa stabilité. On peut évaluer, dans les textes, l'usage du terme en observant son emploi, sa fréquence, sa répartition ou, plutôt, sa récurrence dans plusieurs ouvrages importants. Le terme est compris s'il est connu, s'il fait partie de textes spécialisés. L'usage est donc un critère essentiel de l'efficacité du terme dans la communication. Sans l'usage, le terme ne peut pas être efficace du fait qu'il ne participe pas à la communication.

On sait bien que l'implantation, la fixation des néonymes normalisées — c'est-àdire la manipulation de l'usage terminologique - constitue le problème central de la normalisation et de l'aménagement terminologiques.

Dans les pages précédentes, nous avons expliqué une trentaine de critères, en procédant du plan graphico-phonique au plan lexical. Nous allons maintenant les résumer et les regrouper pour faire ressortir notre appréciation personnelle de leur importance : 
1. La forme et le sens (le concept) d'un terme efficace seront donnés par une définition spécialisée qui sera systémique et, dans la mesure du possible, adéquate par rapport au référent.

2. Le terme efficace sera fixé par l'usage : sa forme et son sens seront donc connus, compris et assumés des usagers.

3. Afin d'être efficace dans la communication, le terme sera employé dans les textes d'une manière non ambiguë ; tout en respectant la liberté d'approche individuelle, on évitera ou signalera toute collision polysémique, homonymique et synonymique.

4. Le terme efficace possédera le degré nécessaire de conformité linguistique, c'est-à-dire de conformité phonique, graphique et morphologique, de maniabilité syntaxique, de correspondance aux types légitimes de formation et de productivité lexicales.

5. L'efficacité du terme sera augmentée par une motivation acceptable : motivation dépourvue d'interférences sémantiques ou émotives, motivation sans conflit entre motif et sens et, si possible, transparente.

6. L'efficacité du terme pourra être accentuée par la facilité phonique et graphique, et par la facilité de mémorisation, d'évocation et de compréhension.

7. Dans un monde où le contact des langues n'est pas un phénomène exceptionnel, les termes efficaces tiendront compte de l'harmonie interlinguistique, tout en respectant l'intégrité de la langue.

8. Malgré la longueur générale des termes, la concision ne devrait pas être écartée en tant qu'instrument possible de l'efficacité terminologique.

L'examen précédent de critères représente une délimitation linguistique qualitative de l'efficacité des termes dans la communication spécialisée. L'emploi de ces critères dépend, toutefois, de facteurs pragmatiques. Il ne peut y avoir de réponse simple ni unique à la question comment faciliter, par des moyens terminologiques, toute communication spécialisée.

Ce que l'on peut faire dans une situation particulière, c'est définir les facteurs pragmatiques de la communication dont il s'agit, et soigneusement comparer ces facteurs avec les critères disponibles. De cette manière, on devrait être à même d'établir un modèle spécial de critères terminologiques.

Un tel modèle pourrait être mis en œuvre dans divers types d'unification terminologique, tels que l'autorégulation ou l'autodiscipline d'un auteur; l'uniformisation d'une terminologie-maison au sein d'un groupe particulier ; la normalisation nationale d'une langue standard; et l'harmonisation interlinguistique. sée.

L'application de tels critères pourrait, en effet, faciliter la communication spéciali-

\section{BIBLIOGRAPHIE}

AUGER, P. et L.-J. ROUSSEAU (1978) : Méthodologie de la recherche terminologique, S.L. : OLF, Encyclopaedia Universalis, 1968, tome 8, "Efficacité et redondance du code ", p. 1010

ERNST, R. (1980) : Dictionnaire de la technique industrielle, tome IV, français-allemand, Wiesbaden : $O$. Brandstetter. (Voir aussi tome IX : français-anglais, 1982.)

FUCHS, M. (1966) : " La langue des sciences ", pp. 523-675, in F. BRUNOT, Histoire de la langue française, $t$. VI, 1re partie, fascicule 2, Paris, A. Colin.

FUNKHOUSER, G.R. et N. MACCOBY (1971) : "Communicating Specialized Science Information to a Lay Audience", The Journal of Communication, vol. 21, March 1971, pp. 58-71.

GREIMAS, A.J. et J. COURTÉS (1979) : Sémiotique. Dictionnaire raisonné de la théorie du langage, Paris, Hachette.

GUIRAUD, P. (1968) : Langage et théorie de la communication, Martinet, 1968, pp. 145-168.

GUIRAUD, P. (1978) : les Mots savants, Paris, PUF. 
GUYTON de MORVEAU et al. (1787) : Méthode de nomenclature chimique, proposée par MM. de Morveau, Lavoisier, Berthollet et de Fourcray, Paris.

HORECKY, J. (1965) : Kritéria terminologie, CČSTCČ, vol. 4, no 4, pp. 193-200.

ISO (1968) : ISO/R 704-1968, Principes de dénomination, Genève, ISO.

KOCOUREK, R. (1979) : " Lexical Phrases in Terminology ", Travaux de terminologie (GIRSTERM), no 1, octobre 1979, pp. 121-153.

KOCOUREK, R. (1980) : la Nature du français technique et scientifique, Ernst 1980, pp. 1153-1174.

KOCOUREK, R. (1982) : la Langue française de la technique et de la science, Wiesbaden et Paris, $O$. Brandstetter et Documentation française.

KOCOUREK, R. (1983) : Rappórts entre la synonymie et la délimitation des notions, TERMIA 1983, pp. 249-265.

MANUILA, A. et al. (1970-1975) : Dictionnaire français de médecine et de biologie, 4 vol., Paris, Masson.

MARTINET, A., éd. (1968) : le Langage, Paris, Gallimard.

MAZUR, M. (1961) : Terminologia techniczna, Varsovie, WNT.

Néologie en marche, série b : Langues de spécialité, nº 2, 1975, Québec, EOQ.

PEIRCE, C.S. (1978) : Écrits sur le signe rassemblés, traduits et commentés par G. Deledalle, Paris.

Répertoire (1982) : Répertoire des avis linguistiques et terminologiques, S.l, ÉOQ.

Répertoire (1983) : Mise à jour du 13 novembre 1982 au $1^{\text {er }}$ octobre 1983, S.l, EOQ.

RICHAUDEAU, F. (1973) : le Langage efficace, Paris, Denoël.

RONDEAU, G. (1981) : Introduction à la terminologie, Montréal, Centre éducatif et culturel.

RONDEAU, G., éd. (1979) : Table ronde sur les problèmes du découpage du terme (Commission de terminologie de l'AILA, août 1978), Montréal, OLF.

TERMIA (1983) : "Problèmes de la définition et de la synonymie en terminologie ", Actes du Colloque international de terminologie, Université Laval, 23-27 mai 1982, S.1., GIRSTERM.

WÜSTER, E. (1970) : Internationale Sprachnormung in der Technik, besonders in der Elektrotechnik (1re éd., Berlin, 1931), Bonn, H. Bouvier.

WÜSTER, E. (1979) : Einführung in die allgemeine Terminologielehre und terminologische Lexikographie, 2 vol., Vienne, Springer. 